\title{
Discriminações condicionais sem conseqüências diferenciais em crianças: efeitos da história de treino precoce de simetria
}

\author{
Sandra Maria Miranda Álvares \\ Grauben José Alves de Assis \\ Isabel Paulino Esteves \\ Maria Elizângela Carvalho Sampaio \\ Universidade Federal do Pará
}

\begin{abstract}
Resumo
O presente estudo avaliou a formação de classes de estímulos através de um treino de duas discriminações condicionais ( $\mathrm{AB}$ e $\mathrm{AC}$ ) com pareamento consistente modelo-comparação correta, sem consequiências diferenciais imediatas e fading, aplicando-se testes de simetria e de equivalência após cada bloco de treino. Participaram quatro crianças do pré-escolar que foram expostas ao procedimento de ensino, em duas etapas: na Etapa 1, com estímulos usuais e na Etapa 2 com estímulos não usuais Para cada modelo, três estímulos de comparação foram apresentados simultaneamente. Cada modelo foi emparelhado consistentemente, com os estímulos de comparação, sendo que o estímulo de comparação correto e o modelo apareceram em fading ao longo do treino. Relações simétricas foram demonstradas com dois participantes na Etapa 1, mas não ocorreram relações emergentes na Etapa 2. Dois participantes transferiram o desempenho obtido de uma etapa para outra com o treino discriminativo, como uma espécie de learning set arbitrário. Os resultados indicam que a sequiência de treino com estímulos usuais e não usuais e a natureza dos estímulos na Etapa 1 foram variáveis relevantes.
\end{abstract}

Palavras-chave: Relações entre estímulos, Pareamento consistente, Ordem de teste, História de treino, Crianças.

\begin{abstract}
Conditional discriminations without immediate differential consequences in children: Effects of training and previous symmetry test history

This study evaluated the formation of stimuli classes through two different conditional relations (AB and $\mathrm{AC}$ ). It used the consistency matching procedure, without immediate differential reinforcement and fading. After each training block, a symmetry or equivalence test was applied. The participants were four pre-school children and were exposed to a teaching procedure divided in two separated steps. At Step 1, usual stimuli were used. And at Step 2, non-usual stimuli were presented. For each sample, three comparison stimuli were appeared at the same time, one of those was consistently matched with a established sample. Both stimuli (correct comparison and sample) were faded during the training. Symmetry was observed in the performances of two participants on Step 1, but there were not emergent relations obtained in Step 2. Two participants transferred the obtained acting in a step to the other, as a type of arbitrary learning set. The results indicate that the sequence of training and tests and the nature of the stimuli at the first step were important variable for successful performance.
\end{abstract}

Key words: Stimuli relations, Consistency training, Test order, Training history, Children.

$\mathrm{U}$ ma importante característica observada em estu dos sobre controle de estímulos é que os organis mos em geral são capazes de formar classes de estímulos. Classes de estímulos podem ser formadas com base na similaridade física entre seus membros, ou através de relações arbitrárias entre estímulos fisicamente diferentes.

Um procedimento tradicionalmente utilizado, em estudos da área, para produzir uma relação de controle condici- 
onal de estímulos tem sido o de matching-to-sample (escolha de acordo com o modelo). Controle condicional ou contextual diz respeito a uma relação entre estímulos, estímulos estes que não controlam diretamente uma resposta, mas que determinam o controle sobre outros estímulos que têm relações específicas com uma determinada resposta, ou seja, certos estímulos irão controlar uma determinada resposta se alguns outros estiverem presentes (de Rose, 1993).

Estudos recentes (Assis, Baptista, Damin \& Álvares, 1997; Assis, Baptista, Kato \& Alves, 2000; Baptista \& Assis, 1995; Barros, Baptista \& Assis, 1998; Damin, Assis e Baptista, 1998; Harrison \& Green, 1990; Williams, Saunders, Saunders \& Spradlin, 1995) têm apresentado resultados positivos sobre os efeitos de variáveis de procedimentos no ensino de relações condicionais em humanos, na ausência de conseqüências diferenciais imediatas. $\mathrm{O}$ uso de um treino com pareamento consistente modelo-estímulo de comparação correto em todas as tentativas, com adultos, préadolescentes e crianças do pré-escolar parece demonstrar alternativas de fontes de controle no ambiente experimental.

Segundo Sidman (1992) nas tentativas em que não haja conseqüências diferenciais imediatas, deve-se programar os arranjos de treino de modo a reduzir as possibilidades de outras fontes de controle de estímulos e assegurar uma única base de escolha, pois a equivalência, segundo ele, pode não ser a única relação possível entre estímulos modelo e de comparação. Sidman (1992) admite, então, a possibilidade de se programarem tarefas experimentais de forma a produzir relações condicionais previsíveis sem consequiências diferenciais imediatas.

Williams et al. (1995) realizaram um estudo que buscava verificar se cinco crianças de idade variando entre 7 e 12 anos, desenvolveriam discriminações condicionais novas após o ensino, sem reforçamento diferencial, de discriminações condicionais com três escolhas. Primeiramente, eles treinaram, com reforço diferencial, as discriminações condicionais $\mathrm{AC}$ ( $\mathrm{A} 1 \mathrm{C} 1, \mathrm{~A} 2 \mathrm{C} 2$ e A3C3) e BC (B1C1, B2C2 E B3C3) e testaram, em seguida, a equivalência (AB e BA). Posteriormente, eles repetiram o mesmo procedimento com novas discriminações condicionais (DF e EF). Todos os participantes desenvolveram 6 classes com 3 membros cada. Contudo, após treinar, reforçando diferencialmente suas respostas, os autores expuseram os participantes a uma situação de discriminação condicional nova, em que os estímulos do conjunto " $C$ " (comparações) eram emparelhados, condicionalmente, com os estímulos do conjunto "D" (modelos), sem reforçar diferencialmente suas respostas. Os resultados indicaram que três dos cinco participantes selecionaram estímulos de comparação diferentes na presença de cada modelo específico. Vale ressaltar que, segundo critérios do experimentador, o requerido era que, individualmente, eles respondessem, de forma consistente, a cada comparação diferente de acordo com um modelo específico. Os testes subsequentes mostraram que 3 classes de 6 estímulos emergiram. Com base nesses resultados, os autores discutiram o desempenho dos participantes como exemplos de resposta condicional generalizada, "learning set" e exclusão.

Segundo Williams et al. (1995), parece que o formato dos treinos sem conseqüências diferenciais garantiram um responder condicional generalizado, uma vez que a posição dos estímulos modelo e de comparação e a reexposição dos participantes a estas situações poderiam ter funcionado como um "learning set" arbitrário, em que qualquer situação nova, apresentada aos participantes, poderia ter ficado sob controle de uma história prévia destes com situações similares, em que o reforço diferencial teria estado presente e, o avanço de uma tentativa para outra, poderia ter se tornado suficiente para manter o responder dos participantes.

Um procedimento de matching arbitrário em que as respostas não foram reforçadas explicitamente, as respostas apropriadas poderiam estar sendo controladas por fatores independentes da contingência específica (a qual seria na presença do modelo, a escolha da comparação positiva), ou seja, poderiam ter ficado sob controle de outras relações mantidas com os demais estímulos (negativos, no caso), ou com outras variáveis (cor, tamanho das figuras etc...); porém, no contexto daquelas novas situações, acabaram por contribuir para um responder relacional com o modelo. Williams et al. (1995) avaliaram que a relação condicional entre os estímulos, naquele contexto em que não houve conseqüência diferencial imediata, poderia também ter ficado sob controle de estímulo por exclusão. Nesse sentido, as situações de exclusão teriam desempenhado um papel na seleção condicional sem reforçamento; sendo, assim, razoável propor que a seleção condicional sem reforçamento e a seleção por exclusão sejam consideradas como duas formas de demonstrar um responder condicional generalizado, e que, ambas parecem contribuir para determinar se as relações demonstradas em testes são condicionais.

Harrison \& Green (1990) já haviam demonstrado a possibilidade de treinar humanos sem reforçar diferencialmente suas respostas, levantando uma questão metodológica quanto à formação de classes de estímulos equivalentes. Os achados dos experimentos com treino sem conseqüência diferencial imediata, além de enfatizarem a importância do necessário cuidado que se deve ter na construção dos testes de equivalência, também sugerem uma crítica à aparente emergência de relações entre estímulos não relacionados diretamente, em testes de alguns estudos de equivalência. Os testes, em alguns casos, poderiam não estar, necessariamente, "testando" relações entre estímulos, mas sim, "treinando" essas relações, uma vez que, variáveis estranhas poderiam 
estar servindo como "sinalizadores" de respostas previsíveis. A validade das inferências realizadas sobre dados experimentais dependeria do controle de variáveis indesejáveis, ao longo de treinos e testes.

Baptista e Assis (1995) apontaram para a importância da natureza dos estímulos e o treino com pareamento consistente como as variáveis responsáveis pelo desempenho positivo, em treinos e teste, de quatro universitários de seu estudo.

Embora a natureza dos estímulos seja relevante na formação de classes com estímulos funcionalmente equivalentes, é importante observar outras variáveis que podem exercer controle sobre o responder (Stikeleather \& Sidman, 1990). Entre outras variáveis envolvidas em procedimentos de treino de discriminação condicional, está a mudança gradual de alguma propriedade de estímulos (fading) e a antecipação dos testes (Damin, Assis \& Baptista, 1998).

Segundo Catania (1998):

...Assim como a resposta que define uma classe operante pode ser gradualmente mudada por procedimentos de modelagem, as propriedades do estímulo que definem uma classe operante discriminada podem ser gradualmente mudadas por procedimentos análogos chamados fading (p. 141)

O fading é um procedimento de ensino que implica na utilização de mudanças graduais no estímulo discriminativo, com o objetivo de promover uma transferência de controle de estímulos e, conseqüentemente, garantir uma aprendizagem mais rápida e sem erro (Catania, 1998; Fields, 1978; Fields, 1979; Fields, 1981; Fields, Newman, Adams \& Verhave, 1992; Sidman \& Stoddard, 1967; Terrace, 1963; Touchette, 1968; Touchette, 1971).

A utilização desse procedimento de ensino requer algumas condições para que seja eficaz. Por exemplo, quando a remoção do primeiro estímulo controle (fading out) e a introdução de um segundo estímulo (fading in) são realizadas de forma não gradual, a transferência da função do controle de um estímulo para o outro pode não ser efetuada (Catania, 1998; Fields, 1981; McIlvane \& Dube, 1992).

Álvares, Baptista e Assis (1995) buscaram analisar o efeito dessa mudança gradual no controle de estímulos, verificando se o treino com pareamento consistente modeloestímulo de comparação correto e fading facilitariam o desempenho de pré-adolescentes em tarefas de matching-tosample (MTS) com estímulos não usuais, na ausência de consequiências diferenciais imediatas. Dos cinco participantes, três alcançaram o critério de acerto e responderam consistentemente aos testes de simetria, porém nenhum aos de equivalência, sugerindo que o treino com pareamento consistente e fading, aliado à antecipação dos testes mereceriam uma investigação mais rigorosa.
Alguns autores como Adams, Fields e Verhave (1993) têm apontado que a ordem de apresentação dos testes pode ser programada do simples-para-complexo ou do complexo-para-simples. Simples-para-complexo consistiria na apresentação de testes mais simples (no caso simetria) para posterior exposição de testes mais complexos (equivalência). Complexo-para-simples corresponderia à apresentação de testes mais complexos (equivalência) para a posterior realização de testes mais simples (simetria). A ordem de realização dos testes parece exercer um controle efetivo no desempenho de participantes humanos. Talvez a antecipação dos testes facilitasse o desenvolvimento de relações condicionais e desempenhos emergentes daqueles participantes da educação infantil, até mesmo porque a antecipação do teste de simetria, por exemplo, poderia funcionar como um mediador para as respostas corretas na exposição aos próximos blocos de treinos sem conseqüência diferencial imediata.

O objetivo do presente estudo foi verificar se a partir da construção de uma história de responder condicional aplicado arbitrariamente, com figuras usuais, em treinos sem conseqüências diferenciais; crianças, em idade educação infantil, passariam a responder condicionalmente e formariam novas discriminações condicionais, quando o procedimento envolvesse estímulos que não fizessem parte da história dada pela comunidade verbal desses participantes, ou seja, figuras geométricas abstratas.

\section{Método}

\section{Participantes}

Participaram quatro crianças, de ambos os sexos, na faixa etária do educação infantil: JES (6 anos e 8 meses), VIT ( 6 anos e 4 meses), ALA ( 6 anos e 10 meses) e AMI (4 anos e 7 meses). Os participantes cursavam a alfabetização (exceto AMI).

Todos os participantes foram recrutados através de contatos pessoais e com a autorização dos pais. Após cada sessão experimental, os participantes ganhavam um lanche e no final do experimento, receberam brindes.

\section{Situação Experimental e Materiais}

As sessões experimentais foram conduzidas em uma sala do Laboratório de Psicologia Experimental da Universidade Federal do Pará, medindo aproximadamente $6 \mathrm{~m}^{2}$. Na sala encontrava-se uma mesa com um microcomputador (IBM-PC-AT DX2/66 Mhz), com um monitor SVGA de tela sensível ao toque; duas cadeiras, uma posicionada de frente para o computador e a outra posicionada ao lado da primeira e, finalmente, uma filmadora instalada atrás do participante. Os estímulos foram apresentados através do monitor de vídeo e o participante respondia tocando, leve- 
mente, a tela sensível, com um dos dedos. Na tela apareciam as "janelas" medindo $4 \mathrm{x} 4 \mathrm{~cm}$ contendo as figuras geométricas abstratas. A apresentação e distribuição dos estímulos nas "janelas", o número de tentativas, a duração da sessão experimental e o registro das respostas corretas e incorretas foram controladas pelo computador, por meio de um programa escrito em linguagem Turbo Pascal (versão 6.0). Todas as sessões experimentais e as entrevistas pósexperimentais foram filmadas, sendo as entrevistas transcritas posteriormente.

No pré-treino foram usados como estímulos não experimentais sinais gráficos de alta resolução gráfica.

Os estímulos foram designados por uma letra e por um número para identificar os membros de uma mesma classe. Códigos alfanuméricos indicaram as relações condicionais, bem como os modelos e respectivos estímulos de comparação. As letras referem-se aos membros das classes de estímulos, e os números às classes previamente programadas.

Foram usados os seguintes conjuntos de estímulos: figuras geométricas consideradas pelos experimentadores como usuais ou familiares aos participantes, isto é, passíveis de serem relacionadas com objetos do cotidiano, ou usuais, na etapa 1 e figuras consideradas como não usuais, ou seja, desconhecidas aos participantes ou estranhas à sua cultura, na etapa 2 (ver Figura 1). No pré-treino, exclusivo para a etapa 1 , os estímulos foram diferentes dos estímulos experimentais (sinais de interrogação, de exclamação, porcentagem e cifrão). Os estímulos eram apresentados através do monitor e localizados na tela, no interior de quatro das cinco "janelas", nas posições central, acima, à direita, abaixo e à esquerda; toda a área das figuras era iluminada, isto é, completamente preenchida. Uma das janelas permanecia desativada. As "janelas" eram retângulos com aproximadamente $4 \mathrm{~cm} \times 5 \mathrm{~cm}$, apresentados com um contorno somente, sendo a cor da parte interna a mesma da tela. Cada estímulo media $3 \mathrm{~cm} \times 3 \mathrm{~cm}$, ficando separado um do outro por $3 \mathrm{~cm}$ e fora designado por uma letra e um número (por exemplo: A1, B2, C3 etc); as letras referiam-se a cada conjunto de estímulos e os números identificavam os estímulos membros de uma mesma classe programada pelo experimentador.

\section{Procedimento}

Foi utilizado um procedimento de emparelhamento com o modelo (matching-to-sample) de três escolhas simultâneas (um modelo e três estímulos de comparação). Foram programadas três fases: pré-treino (Fase 1), exclusivamente para a etapa 1, treino (Fase 2), para as etapas 1 e 2, e testes (Fase 3 ), para ambas as etapas. Todos os participantes que formaram as relações condicionais e as emergentes programadas na etapa 1 eram submetidos, em seguida, à etapa 2 . $\mathrm{O}$ número de sessões experimentais dependia do desempenho de cada participante. Cada sessão teve a duração máxima de trinta minutos.

O pré-treino contou com 18 tentativas. Os estímulos utilizados foram caracteres de alta resolução gráfica (não experimentais) e teve por objetivo familiarizar o participante com o uso da tela sensível ao toque e o procedimento de escolha conforme o modelo.

Na Fase 2 foram programadas as relações condicionais $\mathrm{AB}$ e $\mathrm{AC}$ em blocos de 36 tentativas (ver Quadro 1). Havia um total de nove tipos de tentativas, sendo três tipos para cada modelo. Os treinos mistos eram considerados como linhas-de-base para equivalência e cada um era formado por apenas um bloco de 36 tentativas.

O treino era iniciado com a apresentação apenas do modelo na "janela" central onde permanecia até que o participante tocasse a "janela", produzindo o desaparecimento deste por $1 \mathrm{~s}$, em seguida, o modelo era reapresentado, na mesma posição, juntamente com três estímulos de comparação nas "janelas" laterais. Os estímulos desapareciam após a resposta do participante a uma das janelas laterais, por $1 \mathrm{~s}$, e o mesmo modelo era reapresentado, na mesma posição; após uma nova resposta ao modelo, outra configuração de estímulos era apresentada, e assim por diante, até que fosse completado uma bloco de 36 tentativas. Uma "janela" permanecia desativada (apenas o seu contorno na tela), sendo que qualquer resposta ao modelo, quando acompanhado dos estímulos de comparação, ou à "janela" desativada, não alterava a configuração da tela.

Em todas as tentativas havia um estímulo de comparação considerado positivo ( $\mathrm{S}+$ ), emparelhado consistentemente com o modelo e em fading (ambos com as figuras completamente preenchidas), acompanhados de dois estímulos de comparação negativos. Em cada tentativa com uma determinada configuração, por exemplo: $A 1 B 1+B 2 B 3$, o $S+$ aparecia em todas quatro posições da tela: acima, abaixo, direita e esquerda. A tonalidade da cor azul do modelo e do $\mathrm{S}+$ era gradativamente reduzida (da tonalidade máxima para a mínima), havendo para isso três tonalidades da cor azul: TON 1, TON 2 e TON3. Por conta do balanceamento em cada bloco de treino com 36 tentativas, o número de tentativas em cada tonalidade variou: 12,9 e 6 . Nas tentativas com uma determinada tonalidade (por exemplo, TON 1), o participante deveria apresentar $100 \%$ de acertos para mudar de tonalidades. Caso contrário, o participante era reexposto à aquela tonalidade até 10 vezes, no máximo. Nas nove tentativas finais do bloco de 36 tentativas, o modelo e o S+ eram apresentados sem o preenchimento, isto é, apenas com a linha de contorno da figura (ver Quadro 1).

A escolha do S+ era registrada como correta; a escolha de qualquer um dos S- era registrada como incorreta, pelo computador, embora as respostas corretas e incorretas dos 
Quadro 1-Treino das relações condicionais $\mathrm{AB}$ (A1B1, A2B2, A3B3 e AB misto) e AC (A1C1 A3C3

e AC misto) e testes de Simetria BA e CA e de Equivalência BC e CB.

\begin{tabular}{|c|c|c|c|c|c|c|}
\hline \multirow{2}{*}{ TREINO } & \multirow{2}{*}{ TESTE } & \multicolumn{2}{|c|}{ TIPO DE TENTATIVAS } & $\begin{array}{l}\text { Estímulos do } \\
\text { FADING }\end{array}$ & \multicolumn{2}{|c|}{$\mathrm{N}^{\circ}$ DE TENTATIVAS } \\
\hline & & \multicolumn{3}{|c|}{ RELAÇÃ̃O AB } & FAKCIAL & TOHAL \\
\hline \multirow[t]{18}{*}{ TREINO A1B1 } & & A1 & $\mathrm{B} 1+\mathrm{B} 2 \mathrm{~B} 3$ & TON 1 & 04 & \\
\hline & & A1 & $\mathrm{B} 1+\mathrm{B} 2 \mathrm{~B} 4$ & TON 1 & 04 & \\
\hline & & & $\mathrm{B} 1+\mathrm{B} 3 \mathrm{~B} 4$ & TON 1 & 04 & \\
\hline & & & & & & 12 \\
\hline & & A1 & $\mathrm{B} 1+\mathrm{B} 2 \mathrm{~B} 3$ & TON 2 & 03 & \\
\hline & & A1 & $\mathrm{B} 1+\mathrm{B} 2 \mathrm{~B} 4$ & TON 2 & 03 & \\
\hline & & A1 & $\mathrm{B} 1+\mathrm{B} 3 \mathrm{~B} 4$ & TON 2 & 03 & \\
\hline & & & & & & 09 \\
\hline & & A1 & $\mathrm{B} 1+\mathrm{B} 2 \mathrm{~B} 3$ & TON 3 & 02 & \\
\hline & & A1 & $\mathrm{B} 1+\mathrm{B} 2 \mathrm{~B} 4$ & TON 3 & 02 & \\
\hline & & A1 & $\mathrm{B} 1+\mathrm{B} 3 \mathrm{~B} 4$ & TON3 & 02 & \\
\hline & & & & & & 06 \\
\hline & & A1 & $\mathrm{B} 1+\mathrm{B} 2 \mathrm{~B} 3$ & CONT & 03 & \\
\hline & & A1 & $\mathrm{B} 1+\mathrm{B} 2 \mathrm{~B} 4$ & CONT & 03 & \\
\hline & & A1 & $\mathrm{B} 1+\mathrm{B} 3 \mathrm{~B} 4$ & CONT & 03 & \\
\hline & & & & & & 09 \\
\hline & & & & & & 36 \\
\hline & SIMETRIA B1A1 & B1 & $\mathrm{A} 1+\mathrm{A} 2 \mathrm{~A} 3$ & CONT & 09 & 09 \\
\hline \multirow[t]{17}{*}{ TREINO A2B2 } & & A2 & $\mathrm{B} 2+\mathrm{B} 1 \mathrm{~B} 3$ & TON 1 & 04 & \\
\hline & & & $\mathrm{B} 2+\mathrm{B} 1 \mathrm{~B} 4$ & TON 1 & 04 & \\
\hline & & A2 & $\mathrm{B} 2+\mathrm{B} 3 \mathrm{~B} 4$ & TON 1 & 04 & \\
\hline & & & & & & 12 \\
\hline & & $\mathrm{A} 2$ & $\mathrm{~B} 2+\mathrm{B} 1 \mathrm{~B} 3$ & TON 2 & 03 & \\
\hline & & $\mathrm{A} 2$ & $\mathrm{~B} 2+\mathrm{B} 1 \mathrm{~B} 4$ & TON 2 & 03 & \\
\hline & & $\mathrm{A} 2$ & $\mathrm{~B} 2+\mathrm{B} 3 \mathrm{~B} 4$ & TON 2 & 03 & \\
\hline & & & & & & 09 \\
\hline & & $\mathrm{A} 2$ & $\mathrm{~B} 2+\mathrm{B} 1 \mathrm{~B} 3$ & TON 3 & 02 & \\
\hline & & & $\mathrm{B} 2+\mathrm{B} 1 \mathrm{~B} 4$ & TON 3 & 02 & \\
\hline & & $\mathrm{A} 2$ & $\mathrm{~B} 2+\mathrm{B} 3 \mathrm{~B} 4$ & TON 3 & 02 & \\
\hline & & & & & & 06 \\
\hline & & A2 & $\mathrm{B} 2+\mathrm{B} 1 \mathrm{~B} 3$ & CONT & 03 & \\
\hline & & A 2 & $\mathrm{~B} 2+\mathrm{B} 1 \mathrm{~B} 4$ & CONT & 03 & \\
\hline & & A2 & $\mathrm{B} 2+\mathrm{B} 3 \mathrm{~B} 4$ & CONT & 03 & \\
\hline & & & & & & 09 \\
\hline & SIMETRIA B2A2 & B2 & $\mathrm{A} 2+\mathrm{A} 1 \mathrm{~A} 3$ & CONT & 09 & $\frac{36}{09}$ \\
\hline \multirow{18}{*}{ TREINO A3B3 } & & & $\mathrm{B} 3+\mathrm{B} 1 \mathrm{~B} 2$ & TON 1 & 04 & \\
\hline & & & $\mathrm{B} 3+\mathrm{B} 1 \mathrm{~B} 4$ & TON 1 & 04 & \\
\hline & & A3 & $\mathrm{B} 3+\mathrm{B} 2 \mathrm{~B} 4$ & TON 1 & 04 & \\
\hline & & & & & & 12 \\
\hline & & A3 & $\mathrm{B} 3+\mathrm{B} 1 \mathrm{~B} 2$ & TON 2 & 03 & \\
\hline & & & $\mathrm{B} 3+\mathrm{B} 1 \mathrm{~B} 4$ & TON 2 & 03 & \\
\hline & & A3 & $\mathrm{B} 3+\mathrm{B} 2 \mathrm{~B} 4$ & TON 2 & 03 & \\
\hline & & & & & & 09 \\
\hline & & A3 & $\mathrm{B} 3+\mathrm{B} 1 \mathrm{~B} 2$ & TON 3 & 02 & \\
\hline & & & $\mathrm{B} 3+\mathrm{B} 1 \mathrm{~B} 4$ & TON 3 & 02 & \\
\hline & & A3 & $\mathrm{B} 3+\mathrm{B} 2 \mathrm{~B} 4$ & TON 3 & 02 & \\
\hline & & & & & & 06 \\
\hline & & & $\mathrm{B} 3+\mathrm{B} 1 \mathrm{~B} 2$ & CONT & 03 & \\
\hline & & A3 & $\mathrm{B} 3+\mathrm{B} 1 \mathrm{~B} 4$ & CONT & 03 & \\
\hline & & A3 & $\mathrm{B} 3+\mathrm{B} 2 \mathrm{~B} 4$ & CONT & 03 & \\
\hline & & & & & & 09 \\
\hline & & & & & & 36 \\
\hline & SIMETRIA B3A3 & $\mathrm{F}$ & $\mathrm{A} 3+\mathrm{A} 1 \mathrm{~A} 2$ & CONT & 09 & 09 \\
\hline
\end{tabular}




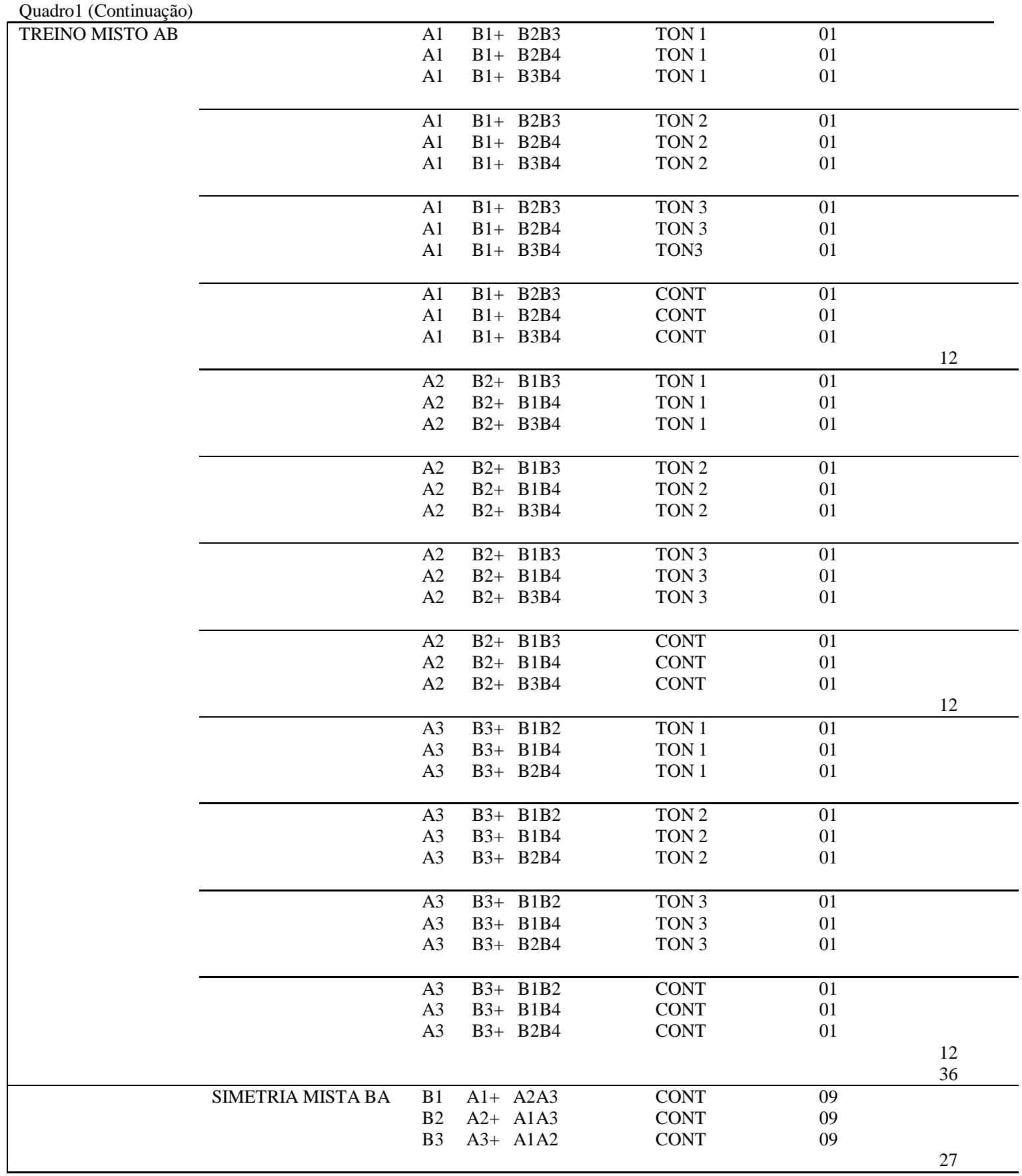


Quadro 1 (Continuação)

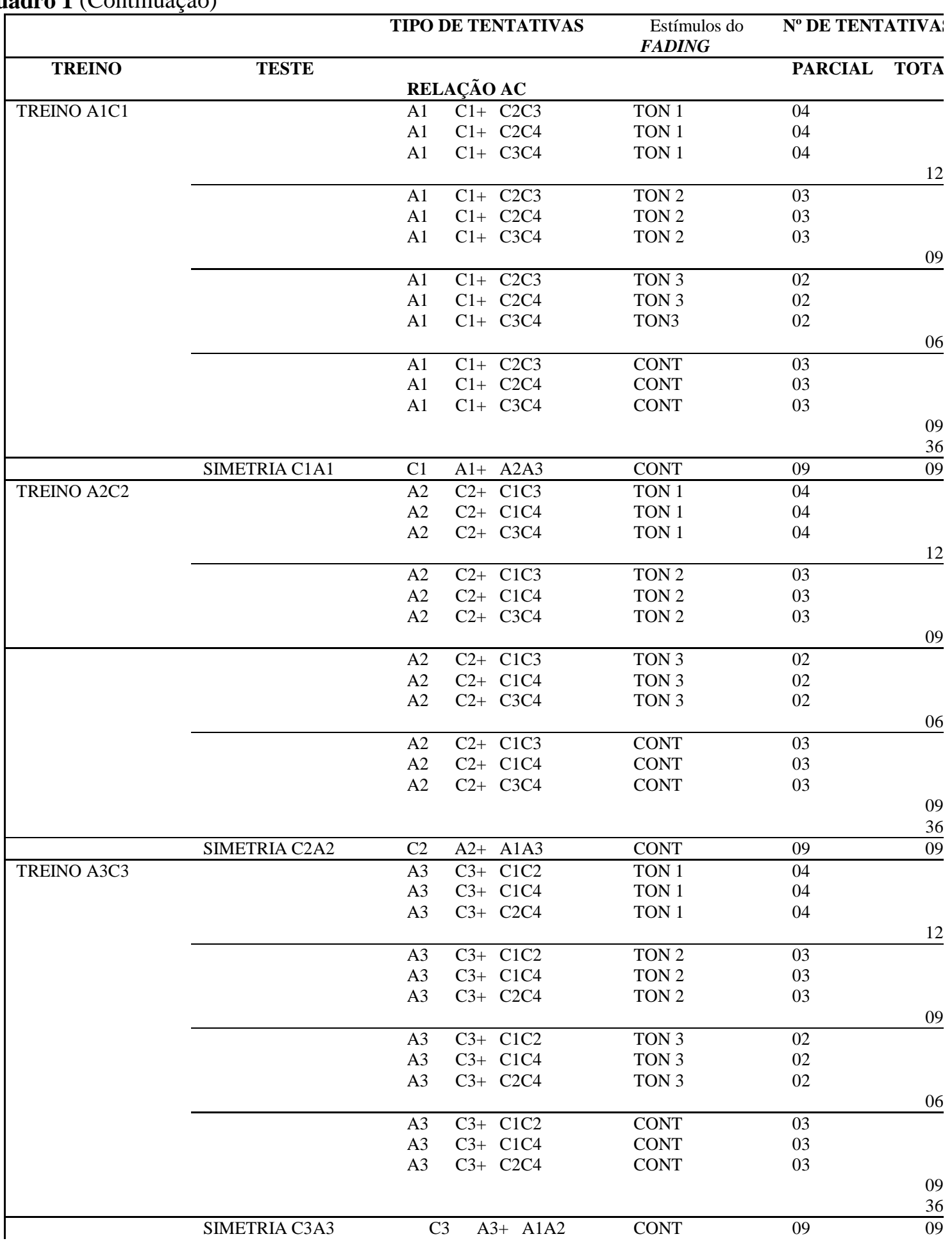




\begin{tabular}{|c|c|c|c|c|c|c|}
\hline \multirow[t]{12}{*}{ TREINO MISTO AC } & & $\begin{array}{l}\text { A1 } \\
\text { A1 } \\
\text { A1 }\end{array}$ & $\begin{array}{l}\mathrm{C} 1+\mathrm{C} 2 \mathrm{C} 3 \\
\mathrm{C} 1+\mathrm{C} 2 \mathrm{C} 4 \\
\mathrm{C} 1+\mathrm{C} 3 \mathrm{C} 4\end{array}$ & $\begin{array}{l}\text { TON } 1 \\
\text { TON } 1 \\
\text { TON } 1\end{array}$ & $\begin{array}{l}01 \\
01 \\
01\end{array}$ & \\
\hline & & $\begin{array}{l}\text { A1 } \\
\text { A1 } \\
\text { A1 }\end{array}$ & $\begin{array}{l}\mathrm{C} 1+\mathrm{C} 2 \mathrm{C} 3 \\
\mathrm{C} 1+\mathrm{C} 2 \mathrm{C} 4 \\
\mathrm{C} 1+\mathrm{C} 3 \mathrm{C} 4\end{array}$ & $\begin{array}{l}\text { TON } 2 \\
\text { TON } 2 \\
\text { TON } 2\end{array}$ & $\begin{array}{l}01 \\
01 \\
01\end{array}$ & \\
\hline & & $\begin{array}{l}\text { A1 } \\
\text { A1 } \\
\text { A1 }\end{array}$ & $\begin{array}{l}\mathrm{C} 1+\mathrm{C} 2 \mathrm{C} 3 \\
\mathrm{C} 1+\mathrm{C} 2 \mathrm{C} 4 \\
\mathrm{C} 1+\mathrm{C} 3 \mathrm{C} 4\end{array}$ & $\begin{array}{l}\text { TON } 3 \\
\text { TON } 3 \\
\text { TON3 }\end{array}$ & $\begin{array}{l}01 \\
01 \\
01\end{array}$ & \\
\hline & & $\begin{array}{l}\text { A1 } \\
\text { A1 } \\
\text { A1 }\end{array}$ & $\begin{array}{l}\mathrm{C} 1+\mathrm{C} 2 \mathrm{C} 3 \\
\mathrm{C} 1+\mathrm{C} 2 \mathrm{C} 4 \\
\mathrm{C} 1+\mathrm{C} 3 \mathrm{C} 4\end{array}$ & $\begin{array}{l}\text { CONT } \\
\text { CONT } \\
\text { CONT }\end{array}$ & $\begin{array}{l}01 \\
01 \\
01\end{array}$ & 12 \\
\hline & & $\begin{array}{l}\text { A2 } \\
\text { A2 } \\
\text { A2 }\end{array}$ & $\begin{array}{l}\mathrm{C} 2+\mathrm{C} 1 \mathrm{C} 3 \\
\mathrm{C} 2+\mathrm{C} 1 \mathrm{C} 4 \\
\mathrm{C} 2+\mathrm{C} 3 \mathrm{C} 4\end{array}$ & $\begin{array}{l}\text { TON } 1 \\
\text { TON } 1 \\
\text { TON } 1\end{array}$ & $\begin{array}{l}01 \\
01 \\
01\end{array}$ & \\
\hline & & $\begin{array}{l}\text { A2 } \\
\text { A2 } \\
\text { A2 }\end{array}$ & $\begin{array}{l}\mathrm{C} 2+\mathrm{C} 1 \mathrm{C} 3 \\
\mathrm{C} 2+\mathrm{C} 1 \mathrm{C} 4 \\
\mathrm{C} 2+\mathrm{C} 3 \mathrm{C} 4\end{array}$ & $\begin{array}{l}\text { TON } 2 \\
\text { TON } 2 \\
\text { TON } 2\end{array}$ & $\begin{array}{l}01 \\
01 \\
01\end{array}$ & \\
\hline & & $\begin{array}{l}\mathrm{A} 2 \\
\mathrm{~A} 2 \\
\mathrm{~A} 2\end{array}$ & $\begin{array}{l}\mathrm{C} 2+\mathrm{C} 1 \mathrm{C} 3 \\
\mathrm{C} 2+\mathrm{C} 1 \mathrm{C} 4 \\
\mathrm{C} 2+\mathrm{C} 3 \mathrm{C} 4\end{array}$ & $\begin{array}{l}\text { TON } 3 \\
\text { TON } 3 \\
\text { TON } 3\end{array}$ & $\begin{array}{l}01 \\
01 \\
01\end{array}$ & \\
\hline & & $\begin{array}{l}\text { A2 } \\
\text { A2 } \\
\text { A2 }\end{array}$ & $\begin{array}{l}\mathrm{C} 2+\mathrm{C} 1 \mathrm{C} 3 \\
\mathrm{C} 2+\mathrm{C} 1 \mathrm{C} 4 \\
\mathrm{C} 2+\mathrm{C} 3 \mathrm{C} 4\end{array}$ & $\begin{array}{l}\text { CONT } \\
\text { CONT } \\
\text { CONT }\end{array}$ & $\begin{array}{l}01 \\
01 \\
01\end{array}$ & 12 \\
\hline & & $\begin{array}{l}\text { A3 } \\
\text { A3 } \\
\text { A3 }\end{array}$ & $\begin{array}{l}\mathrm{C} 3+\mathrm{C} 1 \mathrm{C} 2 \\
\mathrm{C} 3+\mathrm{C} 1 \mathrm{C} 4 \\
\mathrm{C} 3+\mathrm{C} 2 \mathrm{C} 4\end{array}$ & $\begin{array}{l}\text { TON } 1 \\
\text { TON } 1 \\
\text { TON } 1\end{array}$ & $\begin{array}{l}01 \\
01 \\
01\end{array}$ & \\
\hline & & $\begin{array}{l}\text { A3 } \\
\text { A3 } \\
\text { A3 }\end{array}$ & $\begin{array}{l}\mathrm{C} 3+\mathrm{C} 1 \mathrm{C} 2 \\
\mathrm{C} 3+\mathrm{C} 1 \mathrm{C} 4 \\
\mathrm{C} 3+\mathrm{C} 2 \mathrm{C} 4\end{array}$ & $\begin{array}{l}\text { TON } 2 \\
\text { TON } 2 \\
\text { TON } 2\end{array}$ & $\begin{array}{l}01 \\
01 \\
01\end{array}$ & \\
\hline & & $\begin{array}{l}\text { A3 } \\
\text { A3 } \\
\text { A3 }\end{array}$ & $\begin{array}{l}\mathrm{C} 3+\mathrm{C} 1 \mathrm{C} 2 \\
\mathrm{C} 3+\mathrm{C} 1 \mathrm{C} 4 \\
\mathrm{C} 3+\mathrm{C} 2 \mathrm{C} 4\end{array}$ & $\begin{array}{l}\text { TON } 3 \\
\text { TON } 3 \\
\text { TON } 3\end{array}$ & $\begin{array}{l}01 \\
01 \\
01\end{array}$ & \\
\hline & & $\begin{array}{l}\text { A3 } \\
\text { A3 } \\
\text { A3 }\end{array}$ & $\begin{array}{l}\mathrm{C} 3+\mathrm{C} 1 \mathrm{C} 2 \\
\mathrm{C} 3+\mathrm{C} 1 \mathrm{C} 4 \\
\mathrm{C} 3+\mathrm{C} 2 \mathrm{C} 4\end{array}$ & $\begin{array}{l}\text { CONT } \\
\text { CONT } \\
\text { CONT }\end{array}$ & $\begin{array}{l}01 \\
01 \\
01\end{array}$ & $\begin{array}{l}12 \\
36 \\
\end{array}$ \\
\hline \multicolumn{2}{|r|}{ SIMETRIA MISTA CA } & $\begin{array}{l}\mathrm{C} 1 \\
\mathrm{C} 2 \\
\mathrm{C} 3\end{array}$ & $\begin{array}{l}\mathrm{A} 1+\mathrm{A} 2 \mathrm{~A} 3 \\
\mathrm{~A} 2+\mathrm{A} 1 \mathrm{~A} 3 \\
\mathrm{~A} 3+\mathrm{A} 1 \mathrm{~A} 2\end{array}$ & $\begin{array}{l}\text { CONT } \\
\text { CONT } \\
\text { CONT }\end{array}$ & $\begin{array}{l}09 \\
09 \\
09\end{array}$ & 27 \\
\hline \multicolumn{7}{|c|}{ TESTE DE EQUIVALÊNCIA } \\
\hline \multirow[t]{2}{*}{ TREINO MISTOS AB } & EQUIVALÊNCIA & & $\begin{array}{lll}\mathrm{B} 1 & \mathrm{C} 1+ & \mathrm{C} 2 \mathrm{C} 3 \\
\mathrm{~B} 2 & \mathrm{C} 2+ & \mathrm{C} 1 \mathrm{C} 3 \\
\mathrm{~B} 3 & \mathrm{C} 3+ & \mathrm{C} 1 \mathrm{C} 2\end{array}$ & $\begin{array}{l}\text { CONT } \\
\text { CONT } \\
\text { CONT }\end{array}$ & $\begin{array}{l}09 \\
09 \\
09\end{array}$ & 27 \\
\hline & EQUIVALÊNCIA & & 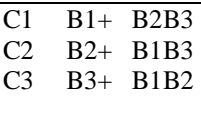 & $\begin{array}{l}\text { CONT } \\
\text { CONT } \\
\text { CONT }\end{array}$ & $\begin{array}{l}09 \\
09 \\
09\end{array}$ & 27 \\
\hline
\end{tabular}

OBS: $-\mathbf{T O N}=$ Tonalidade $\mathbf{C O N T}=$ Contorno das figuras 
participantes não fossem conseqüenciadas diferencialmente. Como critério de aprendizagem, era exigido $100 \%$ de acertos para a passagem de um bloco de treino para outro e aos blocos de testes. Se não atingisse o critério estabelecido no treino, o participante era reexposto ao mesmo até 10 vezes, no máximo. Persistência no erro, após essa máxima reexposição, implicava na apresentação do bloco do treino seguinte.

Foram programados testes de simetria BA e CA, e de equivalência $\mathrm{BC}$ e $\mathrm{CB}$. Cada um aplicado duas vezes no máximo. Uma revisão da linha-de-base (reapresentação de dois blocos de treino misto) era realizada, antes da apresentação dos blocos de teste, se o participante mantivesse o desempenho previsto como critério de aprendizagem. Contudo, se o participante não alcançasse esse critério, este era reexposto aos blocos cinco vezes, no máximo. Se persistisse no erro, após essa re-exposição máxima, o teste deixava de ser aplicado e o participante era submetido ao treino misto seguinte, e assim sucessivamente.

Todos os participantes receberam as seguintes instruções, no início do estudo:

Você terá que descobrir o segredo do jogo. Quando você "achar" que está acertando,

continue jogando até o final. Procure associar duas figuras, ou seja, formar parzinhos. O resultado você saberá no final.

As sessões eram intercalados com jogos e/ou atividades lúdicas visando manter a participação das crianças, nas tarefas experimentais.

Ao final de cada sessão experimental, uma mensagem aparecia na tela: "PARABÉNS, VOCÊ CHEGOU AO FINAL DAS ATIVIDADES. CHAME O EXPERIMENTADOR. MUITO OBRIGADO!".

\section{Resultados}

A Figura 2 apresenta a porcentagem de acerto de todos os participantes. Esses resultados demonstraram que o participante JES, na Etapa 1, com estímulos usuais, atingiu o critério de aprendizagem nos treinos das relações $\mathrm{AB} / \mathrm{AC}$, apresentou as relações emergentes BA/CA; atingiu também o critério nos treinos mistos das revisões de linha de base na Etapa 1 para, em seguida, ser exposto aos testes de equivalência BC e CB, obtendo resultados de 100\%. Na Etapa 2, alcançou o critério de acerto na linha de base, porém não respondeu aos testes de simetria e de equivalência.

O participante VIT alcançou critério de aprendizagem na Etapa 1, no treino das relações $\mathrm{AB} / \mathrm{AC}$; apresentou relações emergentes BA/CA; Em seguida, alcançou o critério na linha de base, respondeu $100 \%$ nos testes de simetria, mas não apresentou equivalência de estímulos. Na Etapa 2 alcançou critério de estabilidade apenas no treino da relação condicional $\mathrm{AB}$, abandonando em seguida o experimento.

O participante ALA, na Etapa 1, alcançou critério exigido nos blocos de treino de aquisição (A1B1, A2B2, A3B3) e testes de simetria B3A3. Atingiu o critério de aprendizagem nos treinos das relações AC e apresentou relações simétricas C2A2 e C3A3; alcançou também o critério de acerto nas revisões de linha de base (treinos mistos $\mathrm{AB}$ e $\mathrm{AC}$ ), não respondeu aos testes de simetria BA e CA, porém alcançou $100 \%$ nos testes de equivalência BC e parcialmente (97\%) no teste CB. Na Etapa 2 alcançou o critério de acerto nos blocos de treino de aquisição (A1B1, A2B2 e A3B3), exceto no último bloco de treino, atingindo resultados de $100 \%$ apenas nas tonalidades a, b, c. No bloco AC alcançou $100 \%$ com todas as tonalidades. Em seguida, apresentou resultados negativos nos testes de simetria BA e de $100 \%$ nos testes de simetria $\mathrm{CA}$; alcançou o critério na revisão da linha de base (treinos mistos $\mathrm{AB} / \mathrm{AC}$ ), no entanto, não respondeu aos testes de simetria e de equivalência.

Um outro participante AMI foi exposto ao treino da relação $\mathrm{AB}$, na Etapa 1, atingindo o critério de acerto nos blocos de treino de aquisição A1B1, A2B2, A3B3 e nos testes de simetria $\mathrm{B} 3 \mathrm{~A} 3$, abandonando em seguida o experimento.

\section{Discussão}

Os resultados mostraram que dois participantes (JES e ALA) expostos ao treino das relações $\mathrm{AB}$ e $\mathrm{AC}$ na Etapa 1 transferiram esse desempenho para a outra condição experimental (2). O participante VIT transferiu parcialmente esse desempenho ao apresentar resultados de $100 \%$ nos blocos de aquisição AB da Etapa 2.

Conforme pode ser observado na Figura 2 nenhum participante apresentou relações emergentes na Etapa 2.

Os resultados demonstraram uma variabilidade inter participantes. Entretanto, parece que o arranjo de treino programado permitiu um controle mais eficaz do que aquele proposto no estudo de Álvares et al. (1995), mas não foi suficiente para reduzir a taxa de evasão. É possível que o atraso no contato do participante com os testes, tenha contribuído para que nenhum dos três tenham alcançado o critério de acerto na linha de base. Em nosso estudo, o contato com as contingências de teste foram imediatas, após cada bloco de treino (ver Quadro 1).

De acordo com o delineamento experimental adotado por Álvares et al. (1995), mesmo quando o participante demonstrasse discriminação condicional, este não era exposto, imediatamente, ao respectivo teste de simetria. Cada participante deveria ser submetido a todos os blocos de treinos para, em seguida, após alcançar a linha de base, ser exposto aos testes. 
Figura 1 - Conjunto de estímulos usado no experimento (estímulos usuais, na parte superior, e não usuais, na parte inferior.

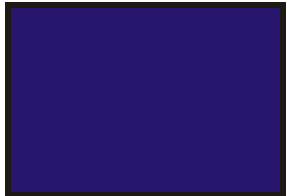

A 1

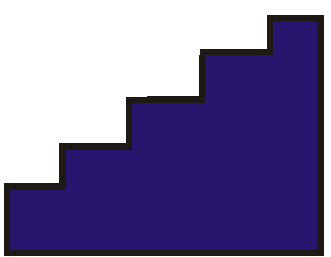

B 1

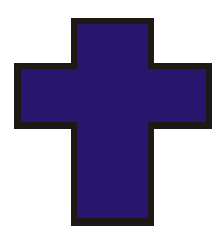

C 1

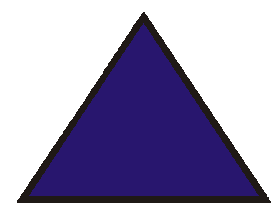

A2

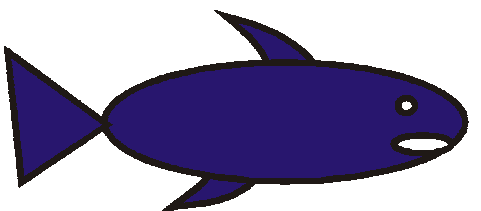

B 2

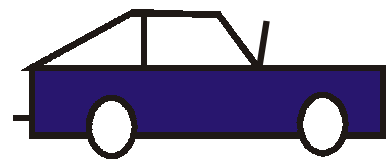

A3

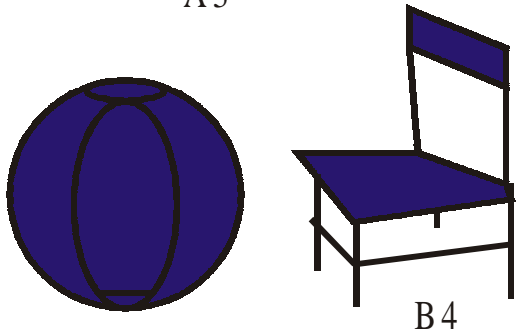

B 3

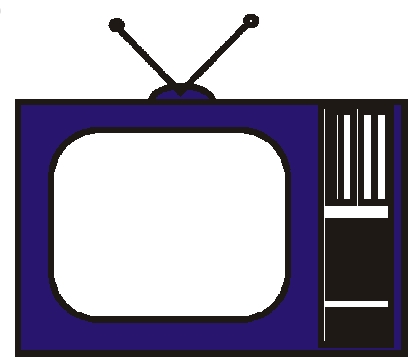

C4

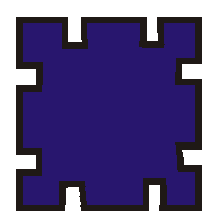

A 1

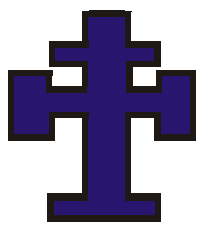

B 1

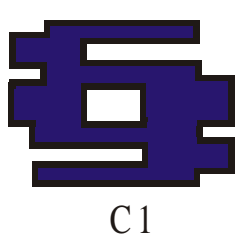

C2

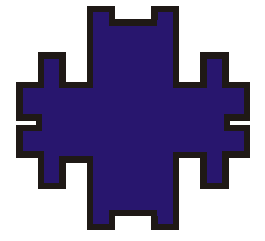

A 2

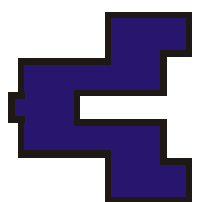

B 2

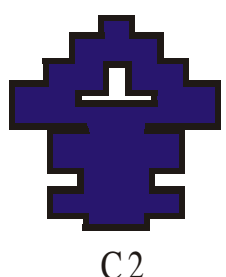

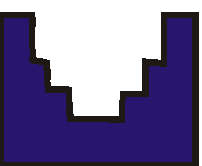

A 3

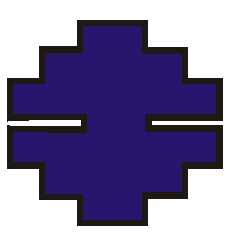

B 3

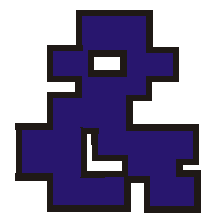

C3

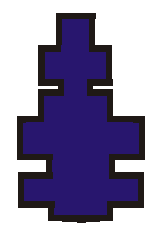

B 4

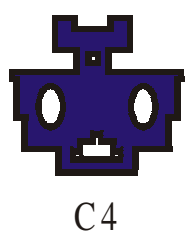


De Rose, Garotti e Ribeiro (1992) usando crianças de uma escola pública, verificaram que a transferência de funções discriminativas em classes de estímulos equivalentes ocorre prontamente. Os autores afirmaram que " os resultados são consistentes com a interpretação de que equivalência de estímulos e equivalência funcional são processos comportamentais distintos, e o primeiro implica o segundo, mas não o inverso" (p. 43).

Os dados aqui apresentados mostram uma pronta transferência do controle de estímulos usuais para não usuais, com o procedimento de treino de pareamento consistente modelo-comparação correta, na ausência de qualquer consequiência diferencial explícita.

Assim, a utilização de um procedimento de treino que avança de uma relação mais simples para uma relação mais complexa (Adams, Fields \& Verhave, 1993), simultaneamente com estímulos usuais, treino com pareamento consistente e fading, parecem assegurar um controle mais efetivo na formação de classes de estímulos em crianças da educação infantil, possibilitando além de um responder condicional generalizado (ver Figura 2, participante JES), uma transferência de controle de estímulos.

Parece, portanto, que a maneira como se organizam os treinos sem consequiências diferenciais poderia estar garantindo um responder condicional generalizado em função de uma história prévia com esses estímulos.

No entanto, por que o alto índice de abandono no estudo de Álvares et al. (1995)? quando três participantes abandonaram o experimento. Vale ressaltar que nos estudos com pré adolescentes e adultos (Assis et al. 1997), envolvendo estímulos usuais e não usuais com longas sessões experimentais, também ocorreu abandono dos participantes. Nossa hipótese é de que, provavelmente, as contingências aversivas impostas, como longas tarefas em extinção, poderiam ter levado ao enfraquecimento da resposta de tocar na tela, produzindo um comportamento tipicamente de extinção (observado, muitas vezes, nos relatos dos participantes).

Outro ponto, que vale ressaltar, é que, no presente estudo, nas situações de teste, em que o fading não estava presente, o desempenho se deteriorou (ver Figura 2), principalmente na Etapa 2 com estímulos não usuais. Parece que o treino, por si só, não garantiu que o participante estivesse respondendo sob controle das relações previstas; evidenciando, desta forma, que, com a retirada da situação de fading, as respostas tenderam a serem emitidas com baixa frequiência, sugerindo, assim, que o participante poderia ter passado a responder aos estímulos sob controle de uma nova contingência, que não a experimentalmente prevista.

Este estudo permitiu evidenciar a natureza dos estímulos como variável relevante na transferência de controle de estímulos, especialmente quando acompanhada de uma mudança gradual dos estímulos (fading) e treino com pareamento consistente (Baptista \& Assis, 1995), produzindo assim um learning set arbitrário (Barros et al., 1998).

Vale ressaltar que estamos adotando o termo transferência (transfer) como usado por Dougher e Markham (1994) enquanto uma aquisição não treinada de função de estímulos, como por exemplo, àquelas estabelecidas nas classes de estímulos equivalentes.

Um número maior de acertos nos treinos discriminativos com crianças do pré-escolar como apresentado neste estudo, representam um ganho no sentido de demonstrar um procedimento de ensino eficaz de relações entre estímulos, na ausência de uma consequiência diferencial imediata. Isto porque, as dimensões dos estímulos, observadas neste estu-

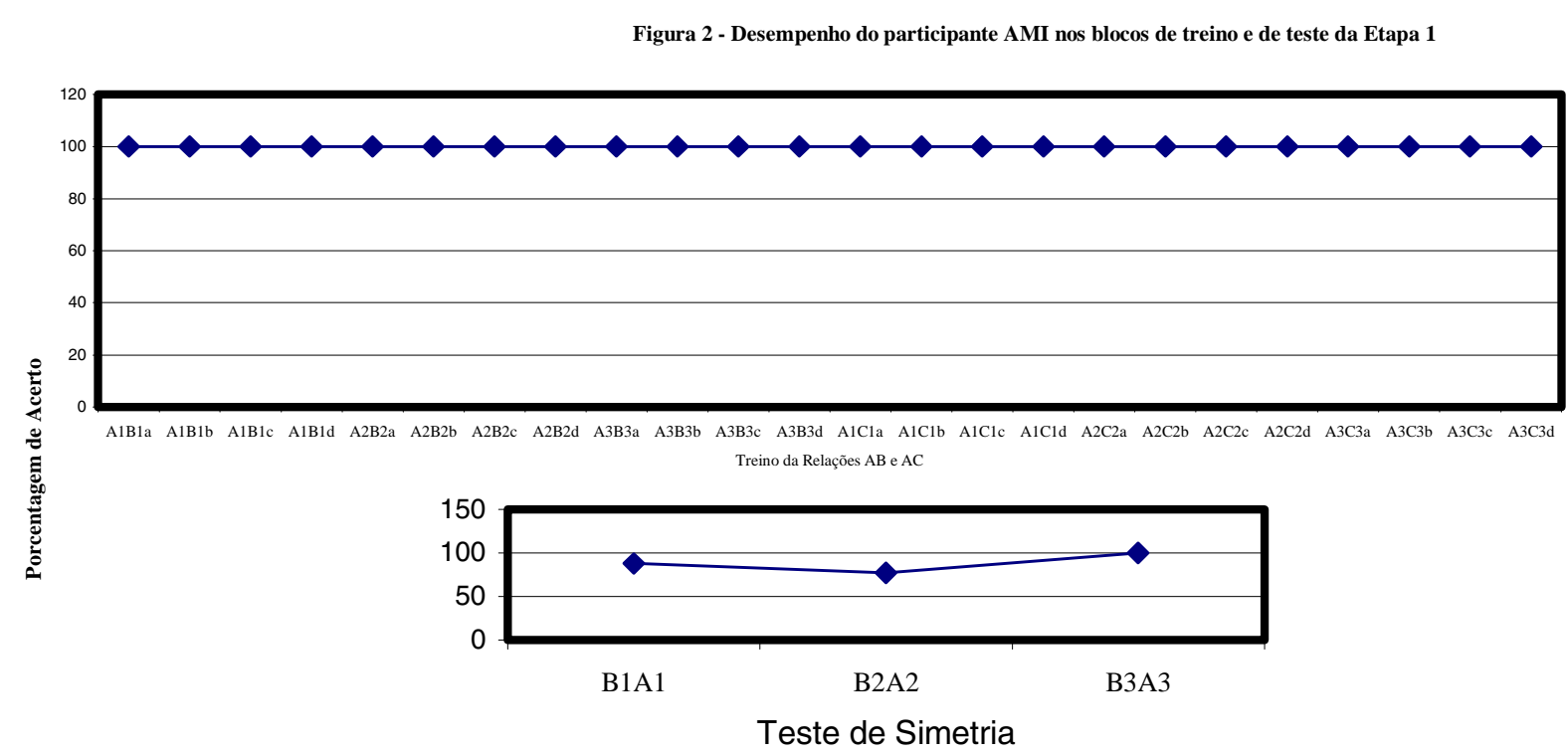


do, sugerem que podemos ensinar relações entre estímulos com um feedback apenas visual (fading).

No estudo relatado por Williams et al. (1995) os autores sugerem que o termo "sem consequiência" parece pouco qualificado para tratar esses procedimentos que não utilizam conseqüências mais imediatas. As consequiências existem. No nosso estudo, elas são temporais (quando o participante acerta 36 tentativas consecutivas ele é exposto imediatamente aos demais blocos, entretanto, quando ele comete mais de 1 erro, deveria ser reexposto ao treino naquela relação até 10 vezes consecutivas, o que leva mais tempo). Provavelmente, a antecipação dos testes em ordem de simples para o complexo e estímulos visuais conhecidos, mesmo com crianças da educação infantil, possibilitou um responder condicional generalizado.

\section{Agradecimentos}

Os autores agradecem as sugestões e críticas do professores Olavo de Faria Galvão e Julio De Rose. O software de controle e registro de dados comportamentais foi elaborado por Ane Margareth Monte Verde Silva, a quem agradecemos.

\section{Referências}

Adams, B. J., Fields, L., \& Verhave, T. (1993). Effects of test order on intersubject variability during equivalence class formation. The Psychological Record, 43, 133-152.

Álvares, S. M. M., Baptista, M. Q. G., \& Assis, G. J. A. (1995). Treino discriminativo e equivalência de estímulos sem conseqüências diferenciais, através de um pareamento consistente e fading com crianças. Anais de Comunicações Científicas (p.1312) da $47^{\underline{a}}$ Reunião Anual da Sociedade Brasileira para o Progresso da Ciência, São Luis - Maranhão.

Assis, G. J. A., Baptista, M. Q. G., Damin, E. T., \& Álvares, S. M. M. (1997). Consistency training and equivalence relations without differential consequences. Experimental Analysis of Human Behavior Bulletin, 1(1), 7-8.

Assis, G. J. A, Baptista, M. Q. G, Kato, O. M., \& Alves, K. R. S. (2000). Relações de equivalência após treino com pareamento consistente de estímulos sob controle contextual. Psicologia: Teoria e Pesquisa, 16 (2), 125-133

Baptista, M. Q. G., \& Assis, G. J. A. (1995). Treino por consistência e equivalência de estímulos sem conseqüências diferenciais. Psicologia: Teoria e Pesquisa. 11 (3), 173-179

Barros, C. W. L., Baptista, M. Q. G., \& Assis, G. J. A. (1998). Efeitos da história de treino sobre a formação de classes de estímulos equivalentes. Acta Comportamentalia, 6 (2), 111-128

Catania, A. C (1998). Learning (4 ${ }^{\text {th }}$ edition). Englewood Cliffs: Prentice-Hall.

Damin, E. T., Baptista, M. Q., \& Assis, G. J. A. (1998). Efeitos da distribuição de treino e testes sobre a formação de classes de estímulos equivalentes sem consequencias diferenciais. Psicologia: Teoria $e$ Pesquisa, 14, (1), 41-49

De Rose, J. C. (1993). Classes de estímulos: implicações para uma análise comportamental da cognição. Psicologia: Teoria e Pesquisa, 9, (2), 283-303.

De Rose, J. C., Garotti, M., \& Ribeiro, I. (1992). Transferência de funções discriminativas em classes de estímulos equivalentes. Psicologia: Teoria e Pesquisa, 8 (1), 43-65

Dougher, M. J., \& Markham, M. R. (1994). Stimulus equivalence, functional equivalence, and the transfer of function. In S. C. Hayes, L.J. Hayes, M. Sato \& K. Ono (Orgs.), Behavior analysis of language and cognition (pp.71-90). Reno: Context.

Fields, L. (1978). Fading and erroless transfer in successive discriminations. Journal of the Experimental Analysis of Behavior, 30, 123-128.

Fields, L. (1979). Acquisition of stimulus control, while introducing new stimuli in fading. Journal of the Experimental Analysis of Behavior, 32, 121-127.

Fields, L. (1981). Early and late introduction of probes and stimulus control acquisition in fading. Journal of the Experimental Analysis of Behavior, 36, 363-370

Fields, L. (1985). Reinforcement of probe responses and acquisition os stimulus control in fading procedures. Journal of the Experimental Analysis of Behavior, 43, 235-241.

Fields, L., \& Verhave, T. (1987). The structure of equivalence classes. Journal of the Experimental Analysis of Behavior, 43 (2), 317-332.

Fields, L., Adams, B. J., \& Verhave, T. (1993). The effects of equivalence class structure on test performances. The Psychological Record, 48, 697-712.

Fields, L., Newman, S., Adams, B., \& Verhave, T. (1992). The expansion of classes through simple discrimination training and fading. The Psychological Record, 42, 3-15.

Harrison, R. J., \& Green, G. (1990). Development of conditional and equivalence relations without differential consequences. Journal of the Experimental Analysis of Behavior, 54 (3), 225-237.

McIlvane, W. J., \& Dube, W. V. (1992). Stimulus Control Shaping and Stimulus Control Topographies. The Behavior Analyst, 15 (1), 89-94

Sidman, M.. (1992). Equivalence relations: some basic considerations. In S. Hayes \& L. J. J. Hayes (Orgs.), Understanding verbal relations (pp. 15-27). Reno, Nevada: Context.

Sidman, M., \& Stoddard, L. T. (1967). The effectiveness of fading in programming a simultaneous form discrimination for retarded children. Journal of the Experimental Analysis of Behavior, 10 (1), 3-15.

Stikeleather, G., \& Sidman, M. (1990). An instance of spurious equivalence relations. The Analysis of Verbal Behavior, 8, 1-11.

Terrace, H. S. (1963). Discrimination learning with and without errors. Journal of the Experimental Analysis of Behavior, 6, 1-27.

Touchette, P. (1968). The effects of graduated stimulus change on the acquisition of a simple discrimination in severely retarded boys. Journal of the Experimental Analysis of Behavior, 11, 39-48.

Touchette, P. (1971). Transfer of stimulus control measuring the moment of transfer. Journal of the Experimental Analysis of Behavior, 15, 347-354.

Williams, D. C., Saunders, K. J., Saunders, R. R., \& Spradlin, J. E. (1995). Unreinforced conditional selection within three-choice conditional discriminations. The Psychological Record. 45, 613-627. 
Departamento de Psicologia Experimental/UFPA e Pesquisador 2C do CNPq. Isabel Paulino Esteves é bolsista de Iniciação Científica (PIBIC/CNPq).

Maria Elizângela Carvalho Sampaio é mestranda no Programa de Pós-Graduação em Teoria e Pesquisa do Comportamento na Universidade Federal do Pará (PA).

Endereço para correspondência: Dr. Grauben Assis: Av. Alcindo Cacela, 2268/503, CEP 66.040000, Bairro da Cremação, Belém, PA. Fone/Fax: 55 (91) 211.1662. E-mail: gjaa@ cpgp.ufpa.br 